Check for updates

Cite this: RSC Adv., 2017, 7, 42393

Received 1st June 2017

Accepted 27th August 2017

DOI: $10.1039 / c 7 r a 06117 j$

rsc.li/rsc-advances

\title{
Origin of surface potential in undoped zinc oxide films revealed by advanced scanning probe microscopy techniques
}

\begin{abstract}
Ting Su (1D) *
This paper presents recent studies combining piezoresponse force microscopy and Kelvin probe force microscopy to reveal the origin of bias-induced changes of surface potential in undoped zinc oxide ( $\mathrm{ZnO}$ ) films at the nanoscale. Surface potential under dc bias in $\mathrm{ZnO}$ films is studied using Kelvin probe force microscopy (KPFM). In addition, the piezoelectric properties of the $\mathrm{ZnO}$ are investigated by applying voltage using piezoresponse force microscopy (PFM), which is adopted to provide new physical insight into the important role of polarization charge in surface potential. PFM and KPFM results suggest that the origin of surface potential under an electric field in undoped $\mathrm{ZnO}$ film is determined by polarization charge, injection charge and surface charge. In addition, significant change in the Fermi level (work function) of undoped $\mathrm{ZnO}$ is found by positive (negative) biasing. The qualitative and quantitative study of surface potential and charge trapping properties can be useful for designing advanced nanoscale nonvolatile memory devices.
\end{abstract}

\section{Introduction}

Zinc oxide (ZnO) has unique electrical, piezoelectric, optical, and ferroelectric-like properties. $\mathrm{ZnO}$ is attracting an increasing level of interest as a direct-band-gap semiconductor for its functional applications. Reprogrammable nonvolatile memory represents an essential element in most modern electronic devices. ${ }^{1} \mathrm{ZnO}$ is a promising material for nonvolatile random-access-memory applications ${ }^{1}$ because it can provide high performance as well as low power consumption, high environmental stability, and optical transparency. The arrangement of the dipoles ${ }^{2}$ and switching features significantly influence macroscopic properties. However, the nanoscale behavior of the $\mathrm{ZnO}$ is not sufficiently understood to make full use of this material.

At the nanometer scale, microscopy ${ }^{3-5}$ techniques have become primary tool for studying surface topography, structure and electronic, mechanical, optical and transport properties. Piezoresponse Force Microscopy (PFM) and Kelvin Probe Force Microscopy (KPFM) is widely used due to good spatial resolution and easy manipulation for polarization..$^{6-8}$ PFM provides additional important information on polarization through local piezoresponse. ${ }^{7}$ When dc bias is applied to the $\mathrm{ZnO}$ sample, polarization switching occurs and screen charge ${ }^{7}$ is transferred to the surface. The polarization charges are of great significance for the origin of surface potential in undoped $\mathrm{ZnO}$ films. Understanding the surface potential under dc bias in the $\mathrm{ZnO}$ films is important for practical application. In addition, it is

State Key Laboratory of Surface Physics, Department of Physics, Fudan University, Shanghai 200433, China. E-mail: 14110190063@fudan.edu.cn interesting to explore how charge trapping effect and polarization charge constitute surface potential. ${ }^{8}$ It has been reported the correlation between polarization charge and surface potential of ferroelectric materials by Kalinin and Kim et al. ${ }^{6-8}$ while the publication on surface potential of polarized regions of the undoped ZnO film remains rare. A report that the surface potential of the zinc oxide film has been studied without external stimuli of electric filed. ${ }^{9}$

However, in previous work, ${ }^{9}$ polarization information can not be obtained. Polarization information is essential to getting new physical insight into the role of polarization charge in surface potential. To study the polarization switching, PFM should be adopted. Therefore, in this study, the origin of surface potential under dc bias in undoped $\mathrm{ZnO}$ was investigated by a combined use of PFM and KPFM. It is found polarization charge is key role in surface potential of undoped $\mathrm{ZnO}$ film. In addition, it is also found Fermi level of undoped $\mathrm{ZnO}$ shifts upward (downward) by positive (negative) bias. The study of trapped charges is promising for zinc oxide based nonvolatile memory application.

The article is organized as follows. In Section 2, material preparation and experimental techniques are described. In Section 3, we present the results and discussion of PFM and KPFM imaging of the undoped $\mathrm{ZnO}$ sample. The conclusion of the article is given in Section 4.

\section{Experiment}

Pulsed laser deposition was adopted to prepare pure $\mathrm{ZnO}$ film on $\mathrm{Si} / \mathrm{SiO}_{2} / \mathrm{Ti} / \mathrm{Pt}$ substrate at $600{ }^{\circ} \mathrm{C}$. Pt is used as bottom 
electrode. The thickness of the sample was measured to be $69 \mathrm{~nm}$. In this work, the thickness of undoped $\mathrm{ZnO}$ thin film was measured by ellipsometry. The popular optical technique for studying the thickness of thin films is ellipsometry, which measures polarization change. Kelvin probe force microscopy is noncontact technique (Fig. 1) to study structural and electrical properties of surfaces and interfaces, and it can obtain nanoscale mapping of surface potential. ${ }^{10}$ KPFM technique exploits long-range electrostatic interactions between sample and tip. In KPFM measurements of conductive samples, tip-sample interaction is only due to Fermi level alignment, therefore, the local surface potential difference is simply related to the tip-sample work function difference. ${ }^{11,12}$ In order to minimize cross talk between the topography and electrostatic signal, two-pass technique is used in KPFM. ${ }^{\mathbf{1 3 , 1 4}}$ In the first pass, topography information is recorded. In the second pass, the tip is lifted to a user-selected separation between sample surface and tip. In addition, several scanning parameters can be controlled in order to optimize the operating condition. KPFM experiments were carried out under an ac voltage of $3 \mathrm{~V}$. The tip is kept a distance of $40 \mathrm{~nm}$ above the sample surface. These scanning parameters are equal to that of the previous work. ${ }^{9}$ A dc voltage $V_{\mathrm{dc}}$ and an ac voltage $V_{\mathrm{ac}} \sin (\omega t)$ are applied to the cantilever, with $\omega$ close to cantilever resonance frequency. ${ }^{10}$ The cantilever oscillated at frequency $\omega$ due to electrostatic force between tip and sample. The $\omega$ component of electrostatic force is given by

$$
F_{\omega}=-\frac{\partial C}{\partial z}\left[\left(V_{\mathrm{CPD}}-V_{\mathrm{dc}}\right) V_{\mathrm{ac}} \sin (\omega t)\right]
$$

where $z$ is the distance between sample and tip, $C$ is the capacitance, $V_{\mathrm{CPD}}$ is the contact potential difference. Moreover, charge trapping properties of the undoped $\mathrm{ZnO}$ films were studied by applying voltage biases. Low scan rate of $1 \mathrm{~Hz}$ is used in this paper, which gives sufficient time to accumulate trapped charge. ${ }^{15}$ The measured surface potential of polarized region is determined by the relationship among polarization, injection charge and surface charge.

Complementarily, piezoresponse force microscopy was used to provide additional important information about local polarization by recording electromechanical displacements. ${ }^{\mathbf{1 6}}$ PFM works according to the reverse piezoelectricity principle, i.e. piezoelectric material will deform in accordance with the applied biases. ${ }^{17}$ PFM is a contact mode and practical technique for local nanoscale imaging, spectroscopy and manipulation of piezoelectric and ferroelectric materials. The PFM tip is brought

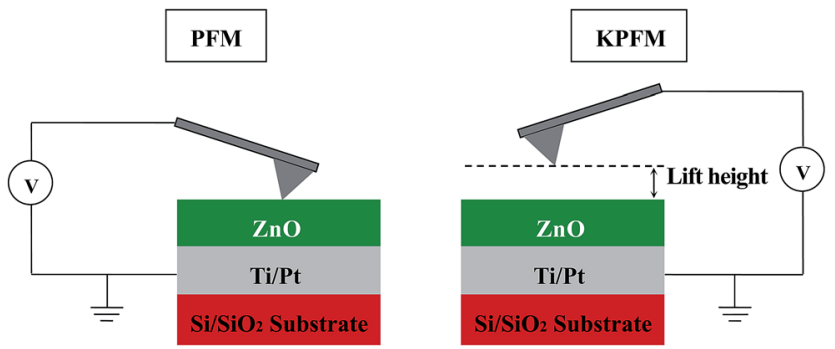

Fig. 1 PFM operating in contact mode and KPFM operating in noncontact mode. into contact with the sample surface during the scan. Fig. 1 shows schematics of the PFM technique. Dual frequency resonance tracking technique ${ }^{18}$ (DART) was adopted in PFM to track the contact resonance. To improve signal-to-noise ratio and magnify the signal, dual excitation of the cantilever independently measures both the amplitude and resonance frequency of the cantilever. The PFM and KPFM measurements were conducted on a commercial SPM (MFP-3D, Asylum Research, USA). A Pt-coated tip (Electri-Lever, Olympus, Japan, with spring constant $=2 \mathrm{~N} \mathrm{~m}^{-1}$ and resonant frequency $=70 \mathrm{kHz}$, tip radius $\sim 15 \mathrm{~nm}$ ) was used. The nature of tip such as its radius, stiffness and sharpness are of great importance to highresolution SPM studies. ${ }^{13,19}$

\section{Results and discussion}

Surface potential of undoped $\mathrm{ZnO}$ film was measured before bias application. Fig. 2(a) and (b) show topography and surface potential images of the $\mathrm{ZnO}$ film respectively. The positive surface potential value suggests the formation of Schottky contact between the $\mathrm{ZnO}$ and metal tip. To explain observations about the metal/semiconductor interface, we consider MottSchottky model. Schottky junctions are characterized by Schottky barrier, depletion width and rectifying behavior. A depletion layer (diffusion layer) and band bending is formed. The KPFM results accurately explain electrical properties of the metal/semiconductor interface. ${ }^{20}$ When the $\mathrm{ZnO}$ sample and the Pt-coated tip are electronic contact, the difference in work function results in charging of the sample and the tip, leading to the contact potential difference. The value of the contact potential difference is $359 \mathrm{mV}$. KPFM is sensitive to the electric potential variations induced by charges locate both at the surface and in the interior of the film. ${ }^{21}$ The surface shown in Fig. 2(a) has roughness rms of $4.9 \mathrm{~nm}$. It has been reported that novel stabilization mechanism on polar surface, which is important discovery. ${ }^{22,23}$ Furthermore, the study of manipulation of subsurface donors in $\mathrm{ZnO}$ provides interesting insight into the understanding of microscopic mechanism about memory effect. ${ }^{24}$

The charge trapping properties of undoped $\mathrm{ZnO}$ film was investigated by studying surface potential under dc bias. A series of negative and positive dc biases, ranging from -3 to $0 \mathrm{~V}$ with a step $1 \mathrm{~V}$ and 0 to $10 \mathrm{~V}$ with a step $2 \mathrm{~V}$, were applied to the undoped $\mathrm{ZnO}$ by contact mode, which procedure is similar to the previous report by Kim et al. ${ }^{6}$ Surface potential image obtained after bias application is shown in Fig. 3(a). In addition, the quantitative data of surface potential under dc bias is shown in Fig. 3(c). For the $\mathrm{ZnO}$ film, positive bias up to $+10 \mathrm{~V}$ results in surface potential change of $56 \mathrm{mV}$. For the negative bias down to $-3 \mathrm{~V}$, surface potential change is $-59 \mathrm{mV}$. Surface potential value of the $\mathrm{ZnO}$ film decreases with the applied negative bias and increases with the applied positive bias. To rationalize the phenomenon, we consider screening $\operatorname{model}^{25}$ for the surfaceinterface junction. After the poling process, screen charges including the charge from the air and injection charge significantly affect the surface potential. In the case of the surface potential under dc bias in $\mathrm{ZnO}$ film, screen charges and 
(a)

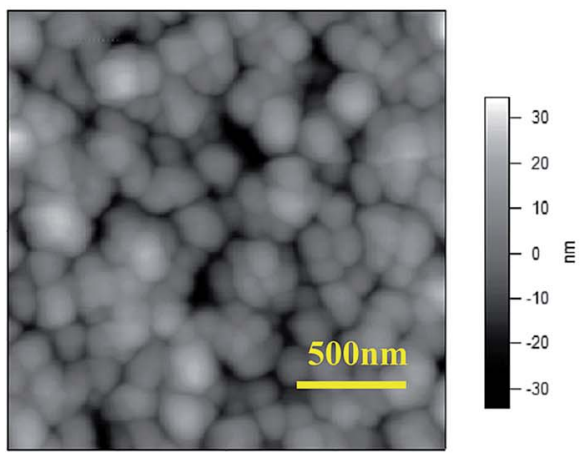

(b)

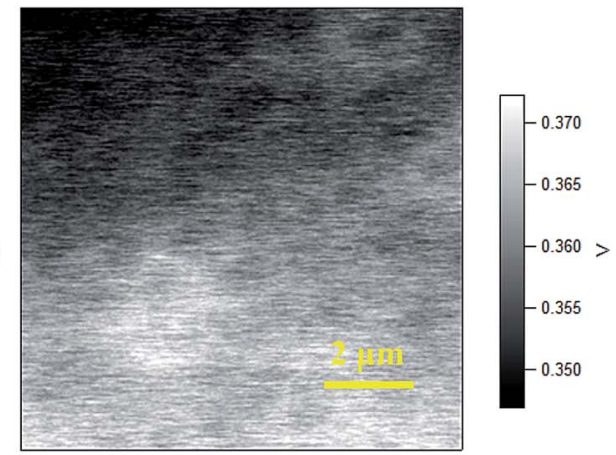

Fig. 2 (a) Surface topography (b) surface potential image of undoped $\mathrm{ZnO}$ film before applying bias.

(a) Surface potential

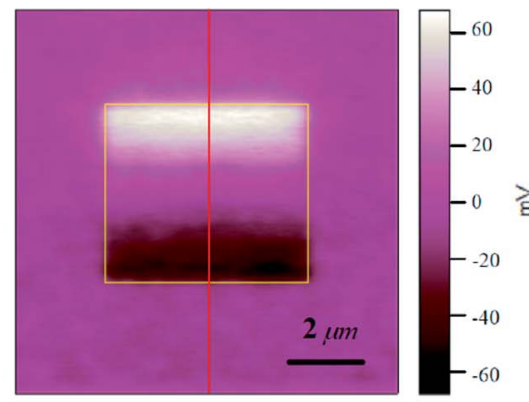

(b)

PFM

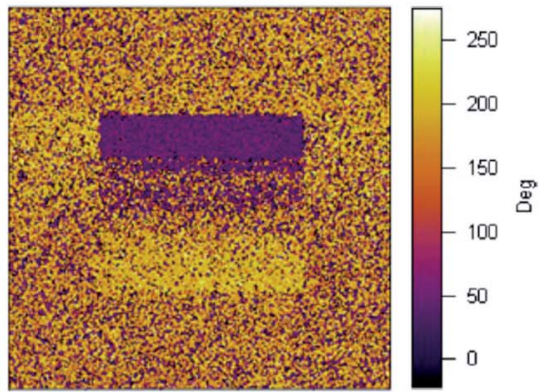

(c)

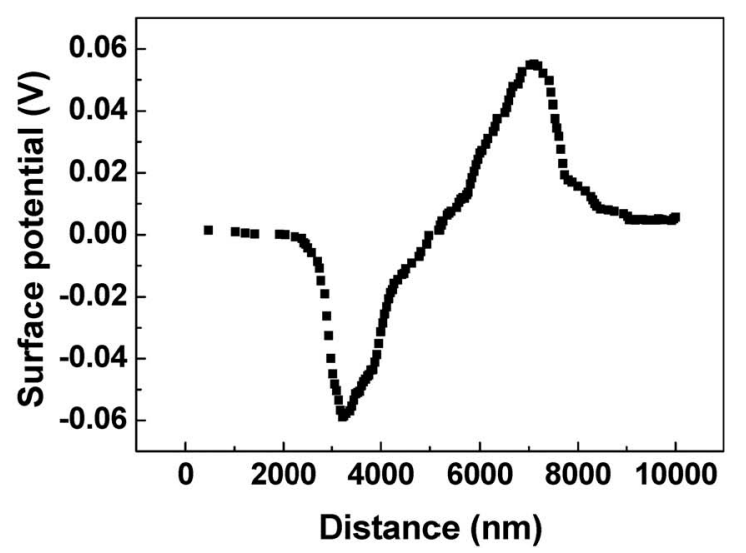

Fig. 3 (a) Surface potential image of undoped $\mathrm{ZnO}$ film after applying bias. (b) PFM phase image scanned on the same region as the surface potential image. (c) KPFM data obtained from (a).

polarization charge contribute to surface potential. Major contribution to surface potential under dc bias in undoped $\mathrm{ZnO}$ film is polarization charge, injection charge and surface charge. Surface potential value can be influenced by the details of sample preparation and synthesis. In addition, it is found Fermi level of undoped ZnO film shifts upward (downward) by positive (negative) bias, as shown in Fig. 4. The Fermi level ${ }^{26}$ of a material is a fundamental quantity that determines its electronic properties. Fermi level position shifts suggest that the electronic structure of $\mathrm{ZnO}$ films change by applying voltage. It is also found $\mathrm{ZnO}$ film is capable of trapping both positive and negative charges, which is promising for nonvolatile memory applications. The charge trapping abilities of undoped $\mathrm{ZnO}$ films may be attributed to oxygen vacancy. Oxygen vacancies ${ }^{27}$ in $\mathrm{ZnO}$ are known to determine physical and chemical properties.

From surface potential data, surface charge density can be calculated by parallel capacitor model. ${ }^{2}$ The capacitance $C$ between sample and tip is expressed as 


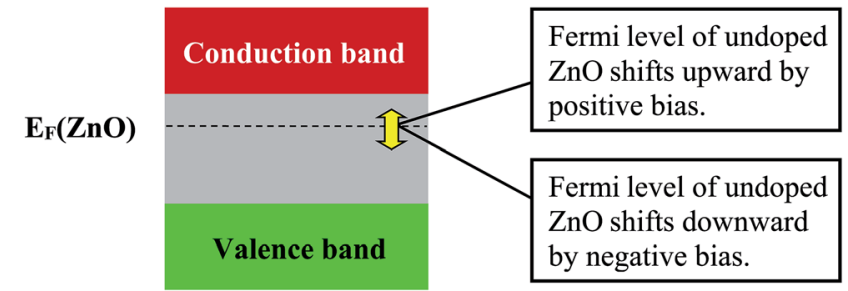

Fig. 4 Schematic band diagram of the $\mathrm{ZnO}$ and it under positive/ negative bias

$$
C=\varepsilon_{0} \varepsilon_{\mathrm{r}} \frac{A}{d}
$$

where $d$ is the thickness of the film, $A$ is the area. The amount of surface charge on the capacitor is

$$
Q=\sigma A=C V
$$

As a result, surface charge density $\sigma$ can be obtained. ${ }^{2}$

$$
\sigma=\varepsilon_{0} \varepsilon_{\mathrm{r}} \frac{V}{d}
$$

Taking the remnant polarization of $0.4 \mu \mathrm{C} \mathrm{cm} \mathrm{cm}^{-2}$ and relative dielectric constant ${ }^{28}$ of 8.75 . Therefore, surface charge density of undoped $\mathrm{ZnO}$ film is calculated to be $1.9 \times 10^{3}$ to $6.5 \times 10^{3} \mathrm{pC} \mathrm{cm}^{-2}$, suggesting that the overscreen ratio is about $1 \%$.

Polarization switching in undoped $\mathrm{ZnO}$ was studied to provide novel perspective into the role of polarization charge in surface potential. Polarization switching under dc bias was studied by PFM on the same region as the KPFM image. PFM phase image after a series of dc biases is shown in Fig. 3(b). The orientation of dipoles in $\mathrm{ZnO}$ is aligned in both upward (yellow color) and downward direction (purple color). When positive voltage of $+2 \mathrm{~V}$ is applied to the $\mathrm{ZnO}$, partial polarization switching occurs and the nucleation of downward domain is observed. The partial polarization switching arises from the small bias to completely reverse the domains. It is important to understand the typical domain switching behavior. PFM is commonly believed to map the domain with the resolution of a few nanometers. ${ }^{29}$ The difference between PFM signals obtained from different domains on a multi-domain sample will be denoted by 'PFM contrast' ${ }^{29}$ PFM phase image provides information on polarization directions. In addition, the position of domain boundary can be determined by PFM measurements. ${ }^{29}$ Different domains are separated by domain boundary (domain wall). ${ }^{30}$ Therefore, it can be distinguished domains in the PFM images. Moreover, the domain size is $60 \mathrm{~nm}$, which studied by PFM. New domain can nucleate and polarized domain can change their orientation due to external electric field. ${ }^{31}$ For the positive bias from $+8 \mathrm{~V}$ to $+10 \mathrm{~V}$, the domain switching angle is $180^{\circ}$ and complete switched domain was observed, suggesting downward polarization written in $\mathrm{ZnO}$. For the negative bias, the PFM phase image show insignificant change after poling. It should be note that polarization switching may be related to defects, which act as pinning centers. $^{32}$ The defect formation enthalpies ${ }^{33}$ in $\mathrm{ZnO}$ are small and the resulting oxygen vacancy influences the domain switching. ZnO has ferroelectric-like properties, which show the (a)

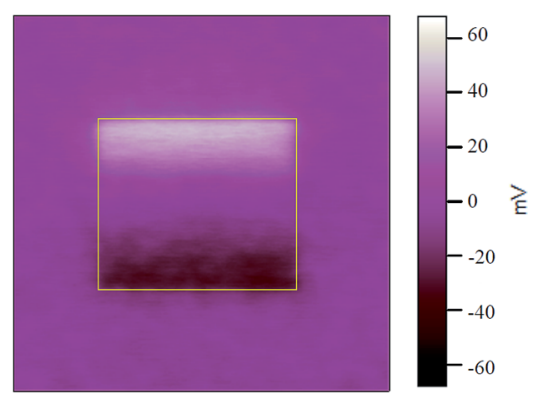

(b)

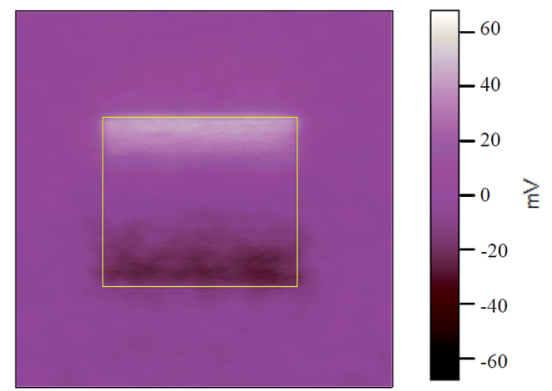

(c)

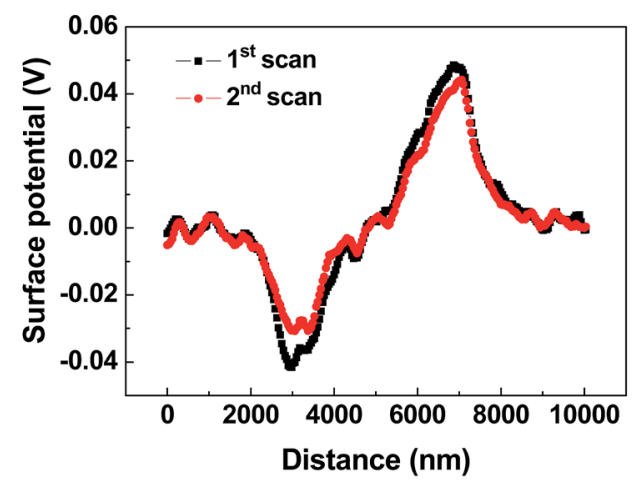

Fig. 5 Surface potential images of undoped $\mathrm{ZnO}$ after (a) the first and (b) second grounded. Tip scan. (c) KPFM data obtained from (a) and (b). 
boundary between unpoled and poled areas. The clearly defined boundary was observed at the area with applied bias of $+8 \mathrm{~V}$ and $+10 \mathrm{~V}$. In addition, as $\mathrm{ZnO}$ has strong piezoelectric response, ${ }^{34}$ mechanical stress could be generated by external electric filed.

The relationship between local polarization orientation and surface potential can be established by comparison of PFM and KPFM image from the same region. ${ }^{7}$ (Fig. 3(a) and (b)) PFM image of the $\mathrm{ZnO}$ film is compared to KPFM image obtained from the same area. The PFM and KPFM results indicate that polarization charge, injection charge and surface charge contribute to surface potential in undoped $\mathrm{ZnO}$ film. The polarization charges are attributed to rotation of the dipoles under dc bias. ${ }^{7}$ Moreover, the stability of trapped holes and electrons in undoped ZnO film was investigated by grounded tip scan, which discharge trapped surface charge. In order to study the role of trapped holes and electrons, discharging experiments were conducted. For the progress of discharging, contact mode scans with grounded tip were carried out for two times, which progress is similar to previous report by Kim et al. ${ }^{6}$ Fig. 5(a) and (b) show surface potential images of undoped $\mathrm{ZnO}$ after the first and second grounded tip scans. The value of surface potential change decreased after grounded tip scans. This observation may be due to the fact that surface charge was discharged. ${ }^{6}$ The PFM and KPFM results reveal that polarization charge, injection charge and surface charge are important for surface potential of undoped $\mathrm{ZnO}$.

\section{Conclusions}

In conclusion, the surface potential of the piezoelectric domains of undoped $\mathrm{ZnO}$ film has been studied by a combined use of PFM and KPFM techniques. We clearly observe piezoelectric properties and surface potential change induced by external electric filed. PFM was adopted to get new information on the relationship between polarization charge and surface potential. We find that polarization charge, injection charge and surface charge play important role in origin of surface potential under dc bias in undoped $\mathrm{ZnO}$ film. In addition, Fermi level position of undoped $\mathrm{ZnO}$ film shifts by positive (negative) bias. Charge trapping properties of holes and electrons suggest that $\mathrm{ZnO}$ is promising for nonvolatile memory applications. Furthermore, the combination of PFM and KPFM is powerful and innovative technique for exploring the origin of surface potential in $\mathrm{ZnO}$ film at nanometer scale, providing novel perspective into physical understanding of advanced surface engineering.

\section{Conflicts of interest}

There are no conflicts to declare.

\section{References}

1 N. Ei-Atab, A. Ozcan, S. Alkis, A. K. Okyay and A. Nayfeh, Appl. Phys. Lett., 2014, 104, 013112.

2 X. Q. Chen, H. Hamada, T. Horiuchi, K. Matsushige, S. Watanable, M. Kawai and P. S. Weiss, J. Vac. Sci.
Technol., B: Microelectron. Nanometer Struct.-Process., Meas., Phenom., 1999, 17, 1930.

3 S. V. Kalinin, R. Shao and D. A. Bonnell, J. Am. Ceram. Soc., 2005, 88, 1077.

4 M. M. Hassan, RSC Adv., 2015, 5, 35497.

5 M. M. Hassan and M. Sunderland, Prog. Org. Coat., 2015, 85, 221.

6 Y. Kim, C. Bae, K. Ryu, H. Ko, Y. K. Kim, S. Hong and H. Shin, Appl. Phys. Lett., 2009, 94, 032907.

7 S. V. Kalinin and D. A. Bonnell, Phys. Rev. B: Condens. Matter Mater. Phys., 2001, 63, 125411.

8 Q. Zhang, C. H. Kim, Y. H. Jang, H. J. Hwang and J. H. Cho, Appl. Phys. Lett., 2010, 96, 152901.

9 T. Su and H. F. Zhang, Chin. Phys. Lett., 2012, 29, 127102.

10 V. Palermo, M. Palma and P. Samori, Adv. Mater., 2006, 18, 145.

11 A. Liscio, V. Palermo and P. Samori, Acc. Chem. Res., 2010, 43, 541.

12 M. Baghar and M. D. Barnes, ACS Nano, 2015, 7, 7105.

13 C. Musumeci, A. Liscio, V. Palermo and P. Samori, Mater. Today, 2014, 17, 504.

14 O. Vatel and M. Tanimoto, J. Appl. Phys., 1995, 77, 2358.

15 J. Y. Son, S. H. Bang and J. H. Cho, Appl. Phys. Lett., 2003, 82, 3505.

16 S. Kalinin, E. Karapetian and M. Kachanov, Phys. Rev. B: Condens. Matter Mater. Phys., 2004, 70, 184101.

17 E. Soergel, J. Phys. D: Appl. Phys., 2011, 44, 464003.

18 B. J. Rodriguez, C. Callahan, S. V. Kalinin and R. Proksch, Nanotechnology, 2007, 18, 475504.

19 G. H. Enevoldsen, T. Glatzel, M. C. Christensen, J. V. Lauritsen and F. Besenbacher, Phys. Rev. Lett., 2008, 100, 236104.

20 O. Vatel and M. Tanimoto, J. Appl. Phys., 1995, 77, 2358.

21 X. M. Lu, F. Schlaphof, S. Grafstrom, C. Loppacher, L. M. Eng, G. Suchaneck and G. Gerlach, Appl. Phys. Lett., 2002, 81, 3215. 22 O. Dulub, U. Diebold and G. Kresse, Phys. Rev. Lett., 2003, 90, 016102.

23 H. Zheng, M. Gruyters, E. Pehlke and R. Berndt, Phys. Rev. Lett., 2013, 111, 086101.

24 H. Zheng, A. Weismann and R. Berndt, Phys. Rev. Lett., 2013, 110, 226101.

25 S. V. Kalinin and D. A. Bonnell, Nano Lett., 2004, 4, 555-560. 26 J. H. Yang, J. S. Park, J. Kang, W. Metzger, T. Barnes and S. H. Wei, Phys. Rev. B: Condens. Matter Mater. Phys., 2014, 90, 245202.

27 D. C. Look, J. W. Hemsky and J. R. Sizelove, Phys. Rev. Lett., 1999, 82, 2552.

28 S. Singh, P. Thiyagarajan, K. Mohan Kant, D. Anita, S. Thirupathiah, N. Rama, B. Tiwari, M. Kottaisamy and M. S. R. Rao, J. Phys. D: Appl. Phys., 2007, 40, 6312.

29 E. Soergel, J. Phys. D: Appl. Phys., 2011, 44, 464003.

30 T. Tybell, P. Paruch, T. Giamarchi and J. M. Triscone, Phys. Rev. Lett., 2002, 89, 097601.

31 D. Y. He, L. J. Qiao, A. A. Volinsky, Y. Bai and L. Q. Guo, Phys. Rev. B: Condens. Matter Mater. Phys., 2011, 84, 024101.

32 S. V. Kalinin, A. N. Morozovska, L. Q. Chen and B. J. Rodriguez, Rep. Prog. Phys., 2010, 73, 056502.

33 P. Erhart, K. Albe and A. Klein, Phys. Rev. B: Condens. Matter Mater. Phys., 2006, 73, 205203.

34 Y. C. Yang, C. Song, X. H. Wang, F. Zeng and F. Pan, Appl. Phys. Lett., 2008, 92, 012907. 